CAHIERS DE

NARRATOLOGIE

\section{Cahiers de Narratologie}

Analyse et théorie narratives

29 | 2015

Street Art 1

\title{
Le genre policier, une ressource narrative de A la recherche de Klingsor de Jorge Volpi
}

\section{Sara Calderón}

\section{OpenEdition}

Journals

\section{Electronic version}

URL: http://journals.openedition.org/narratologie/7455

DOI: 10.4000/narratologie.7455

ISSN: 1765-307X

Publisher

LIRCES

\section{Electronic reference}

Sara Calderón, « Le genre policier, une ressource narrative de A la recherche de Klingsor de Jorge Volpi », Cahiers de Narratologie [Online], 29 | 2015, Online since 31 January 2016, connection on 30 April 2019. URL : http://journals.openedition.org/narratologie/7455; DOI : 10.4000/narratologie.7455

This text was automatically generated on 30 April 2019

Article L.111-1 du Code de la propriété intellectuelle. 


\title{
Le genre policier, une ressource narrative de A la recherche de Klingsor de Jorge Volpi
}

\author{
Sara Calderón
}

1 L'œuvre de Jorge Volpi A la recherche de Klingsor publiée en espagnol en 1999 rapporte l'histoire du lieutenant Francis P. Bacon, physicien chargé par l'armée américaine de découvrir quel éminent homme de science allemand se cache derrière le nom codé de «Klingsor », qui a été attribué au conseiller scientifique secret du Führer ${ }^{1}$. Ecrit sous la forme d'un roman à énigme, le récit introduit un important contenu de divulgation en ce qui concerne la physique quantique. Par ailleurs, il insère également dans la trame l'histoire de l' "Opération Valkyrie », nom de code de la tentative de coup d'Etat portée par plusieurs cadres de l'armée nazie contre Hitler.

2 La voix narrative est assumée par Gustav Links, un mathématicien allemand fictif dont la mission, en sa qualité de témoin et participant aux faits que Bacon doit élucider, est d'épauler ce dernier. Dès le début, le narrateur pose le roman en tant que jeu, régulé par les trois textes intitulés «Lois » qui précèdent chacun des trois livres qui divisent le roman. En effet, les «Lois» assument et revendiquent la subjectivité du narrateur en même temps qu'elles prolongent la fiction vers un paratexte fictionnel qui fait de Gustav Links l'auteur du roman. Après la première « Loi », la diégèse progresse de témoignage en témoignage, entrecoupés du récit des vies des deux personnages principaux, vers la solution de l'énigme.

3 Nous tâcherons de mettre ici en évidence la manière dont le genre policier dans son versant le plus canonique peut intégrer la trame du roman en tant que ressource narrative participant pleinement d'une réflexion sur la connaissance.

\section{Avancées scientifiques et perception du réel}

Dans L'œuvre ouverte, Umberto Eco voyait dans les productions culturelles des « compléments » du monde, c'est-à-dire, des formes autonomes qui se superposaient sur 
lui. En ce sens, il utilisait l'expression "métaphore épistémologique ${ }^{2}$ " pour signifier la manière dont ces formes autonomes étaient déterminées par la façon dont la science permet de percevoir le réel à chaque moment.

Dans le cas des œuvres ouvertes, c'est-à-dire celles sous-tendues par une ambiguïté constituée en valeur affichée et dont le sens dépend de l'intervention active du spectateur ou du lecteur, Eco voyait même l' " écho » de certaines tendances de la science du XXème siècle ${ }^{3}$. En ce sens, il faisait précisément allusion à la question du continuum espacetemps, à l'apparition de l'indéterminé comme catégorie de savoir ou au principe de complémentarité formulé en physique quantique, des avancées scientifiques qui ont permis que le réel soit perçu comme quelque chose d'impossible formulation totale.

Outre le fait qu'il correspond à ce que Eco définissait comme œuvre "ouverte», en ce sens qu'il introduit délibérément en son sein une partie d'incomplétude, A la recherche de Klingsor thématise les principales avancées de la science au début du XXème siècle qui ont abouti à infléchir la perception du réel, réussissant même à les projeter, comme nous le verrons, dans son mécanisme narratif, de façon à renforcer la réflexion que le roman met en place.

7 En effet, dans son enquête sur Klingsor, le lieutenant Bacon s'entretient avec les fondateurs de la physique quantique : Albert Einstein, Max Planck, Werner Heisenberg ou Niels Bohr sont ainsi des personnages du roman. D'autre part, des mathématiciens comme Kurt Gödel, déterminants pour le développement des nouvelles théories, apparaissent aussi dans la trame.

$8 \quad$ Il a été dit que A la recherche de Klingsor est un roman sur le manque de certitudes. Le fait est qu'il reflète aussi le changement de paradigme scientifique qui s'est opéré entre la fin du XIXème siècle et début du XXème et la façon dont celui-ci a pu infléchir la façon dont la société aborde le réel.

Bien que les théories introduites par la trame durant l'enquête de Bacon soient nombreuses, nous en évoquerons ici seulement trois physiques et une mathématique, qui sont celles que l'on peut principalement lier au type de structure du roman et à l'opportune intervention des effets de lecture du récit policier à énigme.

10 Les principales théories qui allaient constituer la base de la physique quantique étaient déjà formulées vers 1935. En 1905 et 1915 successivement, Albert Einstein formule ses théories de la relativité, s'appuyant sur les théories de Max Planck. Il attaque avec elles le postulat d'un espace et d'un temps absolus et rompt ainsi avec la physique newtonienne.

11 D'autre part, c'est en 1927 que Werner Heisenberg formule le principe d'incertitude suivant lequel il est impossible de connaître en même temps la position et la vitesse d'une particule. Prenant pour base ce principe d'incertitude, Niels Bohr, qui avait décrit dès 1913 la structure de l'atome, formule le principe de complémentarité selon lequel la description ondulatoire et la corpusculaire sont toutes deux nécessaires pour comprendre le monde quantique.

12 Enfin, en 1931, Kurt Gödel formule le principe d'incomplétude, suivant lequel tout système axiomatique contient des propositions qui sont tout à la fois vraies et indémontrables, donc, indécidables. Ses théories ont eu d'importantes conséquences sur la façon dont on aborde depuis les mathématiques. 
$13 \mathrm{Au}$ delà des apports scientifiques inestimables, ces théories, formulées en un laps de temps relativement bref, eurent une importante répercussion dans la configuration d'un état du savoir que Werner Heisenberg expose dans ses mémoires :

Peut-être peut-on dire que le progrès de la science exige en général de ceux qui y participent la capacité d'assimiler et d'utiliser des contenus conceptuels nouveaux (...) Cependant, dans une situation où il s'agit réellement de gagner une terre nouvelle, il peut arriver qu'il devienne nécessaire non seulement d'assimiler des contenus nouveaux, mais de procéder à une modification de la structure même de la pensée dès lors que l'on veut comprendre vraiment la situation nouvelle.

En mécanique quantique, on tourne le dos de façon beaucoup plus radicale encore à cet ancien idéal [la description objective]. Ce que nous pouvons transmettre dans un langage objectivant au sens de la physique antérieure, ce sont seulement des affirmations concernant les faits [...] Mais ce qui peut être déduit pour l'avenir de la constatation ainsi faite, cela dépend de la manière dont la question est posée sur le plan expérimental : et cette manière est choisie librement par l'observateur. [...] Mais la prédiction de phénomènes futurs ne peut être faite sans référence à l'observateur ou au moyen d'observation. En ce sens, dans la science actuelle [1927], toute situation physique comporte des aspects objectifs et subjectifs. Le monde objectif de la science du XIXème siècle était, comme nous le savons maintenant, un concept-limite idéal, mais non la réalité. Il sera certes nécessaire également à l'avenir, à chaque confrontation avec la réalité, de distinguer la face objective de la face subjective, de faire une coupure entre ces deux faces ${ }^{4}$.

L'impératif d'abandonner toute certitude auquel se sont confrontés les scientifiques de l'époque est ainsi mis à jour, ainsi que la nécessité de modifier la structure de la pensée à la suite de ces découvertes. Finalement, la connaissance complète de la réalité apparaît identifiée comme un idéal.

Les découvertes de la physique quantique ont contribué, en même temps que les avancées réalisées postérieurement par d'autres sciences, au changement qu'Eco signalait à propos de la manière de percevoir le réel et de celle que l'art a donc pu avoir de le traduire.

C'est de ce point de vue que l'on peut s'interroger sur le sens que peut recouvrir dans un roman tel que $A$ la recherche de Klingsor, qui thématise ces découvertes et se présente à son lecteur imprégné de science jusque dans son langage, le choix du genre policier, et plus particulièrement du roman à énigme, en particulier du point de vue des effets de lecture.

\section{Le pacte de lecture du genre policier}

Les critiques s'accordent à fixer la naissance du roman policier vers le milieu du XIXème siècle, avec la publication par Edgar Allan Poe des canoniques Double assassinat de la rue Morgue (1841), Le mystère de Marie Roget (1843) et La lettre volée (1845). Dans les années qui suivirent, les œuvres de toute une série d'auteurs permirent de fixer les premières conventions du genre: le mystérieux et inexplicable crime d'ouverture, l'importance de l'enquête, le profil du détective, la place prépondérante que prend le raisonnement. Parmi ces auteurs on rappellera Arthur Conan Doyle, qui avec ses romans publiés entre 1887 et 1927 créa le personnage de Sherlock Holmes, qui ne tarda pas à devenir un classique du genre.

Les conventions du genre policier dans la première de ses nombreuses variantes, le roman à énigme, sont ainsi fixées entre la fin du XIXème siècle et le début du XXème. Si 
dans le genre policier l'enquête est primordiale, le roman à énigme se caractérise par une mise en scène particulière du processus rationnel de déduction, à laquelle est subordonné le reste de l'univers fictionnel.

Un premier examen de $A$ la recherche de Klingsor peut assimiler le roman à un roman à énigme, mais un examen plus attentif ne tarde pas à révéler que, si le développement du roman contient certes de nombreuses conventions du genre, celles-ci sont invariablement détournées.

Yves Reuter signale que le genre du policier à énigme est caractérisé par une structure duelle et régressive. En effet, le crime initial s'insère en réalité dans deux histoires : celle qui conduit à le commettre et qui, absente de la trame, détermine cependant la distribution d'indices dans la diégèse, et celle qui rapporte l'enquête du détective ${ }^{5}$.

22 A la recherche de Klingsor remplit en apparence cette convention, puisque le roman débute avec la projection privée pour Hitler des exécutions des principaux intégrants de l'«Opération Valkyrie». Cependant, ce «crime» n'inaugure pas la structure duelle régressive propre au genre policier, car les deux histories ne sont pas en adéquation. En effet, l'enquête rapportée n'est pas associée à ce crime, mais à l'identité et aux crimes de Klingsor qui ne sont introduits par aucun élément concret, même si le lecteur peut en avoir l'intuition de par sa connaissance historique. D'autre part, le lecteur peut connaître par avance l'histoire qui mène à la répression de l' " Opération Valkyrie », qui est celle qui est contée sous la forme d'une analepse.

Le duo détective-assistant est une autre des conventions que le roman de Jorge Volpi reprend seulement en apparence. En effet, le roman à énigme canonique met souvent en scène un duo de détectives: Sherlock Holmes et le docteur Watson; Hercule Poirot et Hastings constituent des exemples emblématiques. Dans ces duos la répartition des rôles est bien définie : le détective est un homme brillant, une sorte de cerveau hypertrophié ou d'esprit absolu caractérisé par ses prodigieuses qualités de déduction auxquelles correspond une importante solitude affective. L'assistant est en revanche un homme un peu ingénu dont les fonctions principales sont de rapporter l'histoire de l'enquête qui mènera à reconstituer l'histoire du crime et de mettre en scène l'étonnement qui doit être celui du lecteur face à la perspicacité du détective ${ }^{6}$.

Dans A la recherche de Klingsor cette distribution de rôles se trouve inversée. En effet, tandis que Gustav Links, le narrateur et assistant, est un homme mûr, solitaire et réfléchi, c'est le lieutenant Bacon qui apparaît caractérisé par son impulsivité et son ingénuité.

La distribution du système indiciaire et des jeux intertextuels avec le genre policier même sont deux autres éléments classiques du policier à énigme que reprend $A$ la recherche de Klingsor. Si les auteurs du policier ironisent souvent à propos des clichés du genre, le narrateur de $A$ la recherche de Klingsor a aussi recours à eux avec malice affirmant avant de commencer l'enquête « (...) el trabajo de detective empezaba a entusiasmarme ${ }^{7}$ ».

Cependant, on pourrait presque dire qu'il y a un double système d'indices. D'une part le lecteur peut trouver les indices distribués par le narrateur concernant les scientifiques interrogés, qui sont destinés à déterminer la culpabilité de Johannes Stark d'abord et de Werner Heisenberg par la suite. D’autre part le lecteur découvre, disséminés dans le récit que fait Gustav Links de sa propre vie et de la conspiration, des indices qui apparaissent sous la forme de lapsus et de contradictions, sur lesquels nous reviendrons.

Signalons pour le moment que, plutôt que de se constituer en roman à énigme, A la recherche de Klingsor intègre une importante composante parodique référée à ce genre ${ }^{8}$. Il 
convient en ce sens de s'interroger sur les effets que la répétition d'éléments marquant l'appartenance générique peut introduire dans le roman.

Le roman policier est apparu dans un contexte où le paradigme scientifique était très différent de celui thématisé par A la recherche de Klingsor et presque pourrait-on dire qu'il surgit dans le sillage de ce paradigme. Denis Mellier parlait à ce propos de «fiction de la raison positive » et il signalait qu'il s'établit dans ce type d'œuvre une lecture qui prend pour modèle le paradigme de la sémiologie médicale et de sciences naissantes telles que la psychanalyse, un "paradigme de lecture du plus petit détail, de la trace infime et résiduelle ${ }^{9}$ ». Si on considère cela, loin de sembler une coïncidence, ou peut-être un choix arbitraire dérivé d'un effet de mode, le genre policier à énigme paraît s'intégrer dans A la recherche de Klingsor comme une pièce du puzzle complexe que le roman met en place, car ce sont précisément les conventions de genre qui incitent le lecteur à aborder le roman avec un certain type de lecture.

Borges signalait que le genre policier avait inventé un type de lecteur, le lecteur qui a des soupçons sur tout et principalement à propos du texte et de son auteur ${ }^{10}$. Compte tenu de cette observation, qui a été depuis lors réitérée par la critique, il convient d'examiner l'effet que produit dans la lecture de A la recherche de Klingsor l'insertion de cette modalité particulière de lecture suspicieuse qui est celle du roman policier à énigme.

\section{La convention générique comme ressource narrative}

Le roman policier naît donc à l'époque du positivisme, caractérisée par une foi profonde en la raison et une volonté de parvenir à la connaissance par le biais de l'expérience scientifique $^{11}$. C'est d'ailleurs le positivisme qui marque durant le XIXème siècle la médecine de son empreinte de recherche de la preuve, créant le modèle de sémiologie médicale dont s'inspirera par la suite le genre policier, en particulier avec Conan Doyle, qui était médecin. En ce sens on peut dire que, par delà les œuvres, le genre policier d'énigme porte aussi la trace d'une façon d'envisager le réel associée à un moment historique.

31 C'est ce trait propre au genre policier le plus classique qui semble être plus particulièrement pertinent pour l'analyse des effets de sens que le dispositif générique peut entraîner dans A la recherche de Klingsor et en particulier de la place qu'il occupe dans la réflexion scientifique et philosophique. En effet, du point de vue de la thématique, le roman aborde une époque où se produit une véritable révolution sur le plan scientifique ainsi qu'un infléchissement tant du paradigme scientifique que de la perception du réel. De ce point de vue, le choix d'un genre empreint d'un paradigme situé à l'opposé de celui que le roman thématise, d'un paradigme basé sur la conviction que l'on peut connaître la totalité du réel et qui désigne l'expérience comme voie préférentielle d'accès à la connaissance, interpelle.

32 En effet, A la recherche de Klingsor induit par son recours aux conventions de genre une lecture qui correspond au pacte de lecture du policier à énigme ; pourtant ce mécanisme ne se met en marche que pour être désactivé presque immédiatement parce que la structure narrative rend impossible la mise en place du jeu logico-déductif, qui constitue l'élément sine qua non du roman à énigme.

33 Les «Lois » qui précèdent chacun des trois livres, formulées en réalisant un pastiche scientifique, énoncent un élément primordial dont le lecteur doit tenir compte dans sa 
lecture du texte: le narrateur ment et il est un criminel. C'est ce qu'établissent principalement les «Leyes del movimiento narrativo» et les «Leyes del movimiento criminal $^{12} »$ :

Durante años se nos ha hecho creer que cuando leemos una novela o un relato escritos en primera persona [...] nadie se encarga de llevarnos de la mano por los acertijos de la trama [...] Mediante este procedimiento, se concibe la ilusión de que un libro es un mundo paralelo en el cual nos internamos por nuestra propia cuenta. Nada más falso. A mí siempre me ha parecido intolerable la mezquindad con la cual un escritor pretende esconderse detrás de sus palabras, como si nada de él filtrase en sus oraciones o sus verbos, aletargándonos con una supuesta dosis de objetividad ${ }^{13}$.

Ce premier paragraphe mobilise les notions d'objectivité et de subjectivité, jouant non sans malice, lorsqu'il situe écriture et narration sur le même plan, avec les postulats structuralistes d'approximation objective du texte ${ }^{14}$. La subjectivité revendiquée d'emblée est affirmée lorsque Gustav Links se définit comme «el guía que habrá de llevarlos a través de este relato (...) que se compromete, desde ahora, a dirigir los pasos de sus lectores ${ }^{15}$ ». Cependant, le glissement sémantique de "guider » à "diriger » n'est pas anodin car il introduit une inquiétude à propos de l'intentionnalité du narrateur, que les lignes suivantes se chargeront de développer. Ainsi, lorsque le discours introduit le message principal contenu dans ces premières Lois, mis en avant par une typographie en italique, l'effet est inquiétant : « La verdad es mi verdad y punto ${ }^{16}$ ».

De l'affirmation évidente et même banale et indiscutable du point de vue, le texte passe imperceptiblement à quelque chose de plus sombre, qui ira en s'affirmant de lapsus en contradictions tout au long de la diégèse pour être précisé dans les deux Lois suivantes. La deuxième Loi revient ainsi sur ces considérations, les appliquant toutefois au domaine bien plus trouble du crime. Ainsi, si les premières lois jouaient avec l'idée du texte en tant que crime, les deuxièmes métaphorisent le crime comme texte :

Un cadáver, una herida o el fracaso ajeno son los textos - las huellas - con los cuales el criminal expresa su concepto del mundo.

Quien es capaz de asesinar, robar o traicionar, no cesará en su intento de justificarse y de establecer, por tanto, su propio índice de verdad sobre los hechos que ha provocado. [...] Todos los criminales están obsesionados por el recuento de sus actividades, tanto o más que aquellos que los persiguen e intentan castigarlos. Sólo que su verdad es otra, elusiva y torva, ajena a la rígida lógica de sus perseguidores ${ }^{17}$.

Les «Leyes del movimiento criminal » reviennent sur les thématiques des «Leyes del movimiento narrativo ${ }^{18} »$. A partir de l'inversion texte comme crime - crime comme texte elles reprennent la réflexion sur la subjectivité, l'imprégnant d'un caractère plus sombre. La typographie en italique, qui avait isolé et précisé le type de "vérité ", réapparaitt ici, cette fois pour mettre en avant une altérité terrible.

Colas Duflo montrait dans son article que, au contraire de ce qu'une appréhension superficielle pourrait laisser penser, le lecteur de romans policiers ne joue pas tant avec les faits qu'avec le langage ; c'est-à-dire, que le jeu du roman policier ne serait pas tant un jeu référentiel avec la réalité qu'un jeu textuel ${ }^{19}$. A la recherche de Klingsor vérifie lui aussi ce type de fonctionnement avec le langage et les mécanismes narratifs. Ainsi, ce que le lecteur peut percevoir de la sombre duplicité du narrateur se construit grâce aux écarts de langage comme ceux que nous avons observés plus haut, des omissions, des parallélismes, voire même des anagrammes. 

plusieurs possibilités d'histoire qui, si elles s'excluent entre elles, n'en constituent pas moins des possibles de lecture, alors le lecteur se voit aussi dans l'obligation de conditionner sa lecture à l'adoption consciente d'un point de vue. Tout en fantasmant avec l'idéal énoncé par Borges dans «Le jardin des sentiers qui bifurquent », le roman projette dans la narration la théorie de Heisenberg suivant laquelle il est impossible de mesurer en même temps deux dimensions, en ce sens que, pour correspondre à notre

Cahiers de Narratologie, 29 | 2015 
expérience du réel, il est impossible d'adopter en même temps deux possibilités d'histoire.

Les conventions du policier induisent la mise en place d'une lecture de A la recherche de Klingsor propre à ce genre, produisant dans la pratique une mise en échec du jeu logicodéductif qui entraîne la remise en cause de l'image de la raison positive qui préside à ce type de fiction. Denis Mellier démontrait que, bien que mettant en scène le fonctionnement d'une raison fantasmée, le mécanisme narratif du policier repose en réalité sur une autonomie textuelle qui rend invisibles les paradoxes qui sous-tendent ces fictions et, surtout, l'illusion qu'à un signifiant correspond un seul signifiée ${ }^{22}$.

C'est ici que se trouve l'autre grande divergence narrative entre A la recherche de Klingsor et un roman policier canonique tel que Le meurtre de Roger Ackroyd. En effet, le roman policier peut être une fiction de la déduction parce que tous ses éléments n'ont finalement qu'un seul sens: celui qu'énonce le détective. A la recherche de Klingsor introduit un dispositif de lecture propre au policier, rendant impossible par la suite pour le lecteur l'invalidation de certains possibles de l'histoire qu'il vient de lire. C'est en ce sens qu'il confronte le lecteur à l'absence de prise à laquelle se sont confrontés les pionniers de la physique quantique et, dans une autre dimension, à l'absence de prise de la raison face à des phénomènes d'horreur collective tels que ceux qui se sont produits sous le Troisième Reich.

49 A la recherche de Klingsor prolonge la pratique narrative déjà mise en place par Volpi dans des œuvres comme A pesar del oscuro silencio ou El temperamento melancólico de projeter dans les aspects formels du roman certains des traits de la thématique. D'autre part, il s'insère dans la tendance de la littérature contemporaine de faire déborder la fiction à des parties de l'œuvre qui longtemps ont été réservées au réel, comme le paratexte, et d'utiliser pleinement toutes les ressources fictionnelles, en ce cas, les conventions de genre pour ce qu'elles symbolisent et impliquent du point de vue lectorial, plus que pour ce qu'elles sont.

En effet, dans un roman à ce point imprégné de science, le recours à un genre d'une projection scientifique si marquée, le roman policier à énigme, apparaît comme une sorte de métonymie introduisant dans le récit la façon d'aborder le réel que la science proposait à un moment donné et qui est sur le point de disparaître dans la période historique que traite le roman.

\section{BIBLIOGRAPHY}

BORGES, Jorge Luis, Ficciones, Madrid, Alianza editorial, 2002 (pub 1944).

CALDERON, Sara, Jorge Volpi ou l'esthétique de l'ambiguïté, Paris, L'Harmattan, 2010.

COLLECTIF, Philosophies du roman policier, Paris, Feuillets de l'ENS Fontanay-Saint Cloud, 1995.

ECO, Umberto, L'œuvre ouverte, Paris, Seuil, 1965. 
HEISENBERG, Werner, La partie et le tout. Le monde de la physique atomique, Paris, Flammarion, 1972.

HUTCHEON, Linda, « Ironie et parodie : stratégie et structure », Poétique, n 36, novembre, 1978, p. 446-477.

HUTCHEON, Linda, «Ironie, satire et parodie. Une approche pragmatique de l'ironie », Poétique, n ${ }^{\circ} 46$, avril, 1981, p. 140-156.

JAMESON, Fredric, El posmodernismo o la lógica cultural del capitalismo avanzado, Barcelona, Paidós, 1995.

LOPEZ DE ABIADA, José ; JIMENEZ RAMIREZ, Félix ; LOPEZ BERNASOCCHI, Augusta, En busca de Jorge Volpi. Ensayos sobre su obra, Madrid, Verbum, 2004.

REUTER, Yves, Le roman policier et ses personnages, Saint-Denis, Presses universitaires de Vincennes, 1989.

REUTER, Yves, Le roman policier, Paris, Nathan Université, 1997.

SCHAEFFER, Jean-Marie, Qu'est-ce qu'un genre littéraire ?, Paris, Seuil, 1989.

TODOROV, Tzevan, « Typologie du roman policier », Poétique de la prose, Paris, Seuil, coll.

«Essais », 1971. P. 9-19.

\section{NOTES}

1. VOLPI, Jorge, A la recherche de Klingsor, traduit de l'espagnol (Mexique) par Gabriel Iaculli, Paris, Plon, 2001.

2. ECO, Umberto, L'œuvre ouverte, Paris, Seuil, 1965, p. 28.

3. ECO, Umberto, L'œuvre ouverte, op. cit., p. 29-31.

4. Werner Heisenberg, La partie et le tout. Le monde de la physique atomique. Souvenirs (1920-1965), Paris, Flammarion, coll. Champs, 1972. Pp. 104-105 ; p. 126.

5. REUTER, Yves, Le roman policier, Paris, Nathan Université, 1997, p. 39-40.

6. REUTER, op. cit., p. 43-45; p.46-48.

7. VOLPI, Jorge, op. cit., p. 183. «Ce travail de détective commençait à m'enthousiasmer », VOLPI, Jorge, op. cit., p. 244.

8. Nous adoptons ici les perspectives développées par Linda Hutcheon, qui attribue à la parodie un «éthos » non marqué. Elle s'éloigne en cela de Gérard Genette, qui attribue à la parodie une fonction ludique. Nous aborderons ici la parodie en tant que "genre hypertextuel », suivant les mots de Jean-Marie Schaeffer, pour sa capacité à mettre en contact deux textes entre lesquels elle installe une distance critique. Linda Hutcheon, "Ironie et parodie: stratégie et structure", Poétique, $\mathrm{n}^{\circ} 36$, novembre 1978, p. 446-447. Et «Ironie, satire et parodie. Une approche pragmatique de l'ironie ", Poétique, n 46, avril 1981, p. 140-156. Pour l'étude de la parodie en tant que genre voir Jean Marie Schaeffer, Qu'est-ce qu'un genre littéraire?, Paris, Seuil, 1989.

9. MELLIER, Denis, «L'illusion logique du policier », Philosophies du roman policier, Paris, Feuillets de l'ENS, 1995. p. 81, p. 82. L'article rend compte de la façon dont le genre crée une illusion logique reposant sur l'ensemble de paradoxes qui informent le texte ainsi que sur l'autonomie textuelle. De cette façon, tout en jouant avec cette illusion de la détection, le genre anticiperait en termes de lecture sur la production à venir (Ulysses de Joyce etc) par son jeu avec les possibles de lecture.

10. Cité par DUFLO, Colas, «Le livre-jeu des facultés : l'invention du lecteur du roman policier », Philosophies du roman policier, op. cit. p. 113-114. L'auteur précise l'idée en disant qu'il s'agit d'un 
lecteur qui soupçonne l'écriture elle-même : «l'invention d'un lecteur qui soupçonne le texte et son auteur », op. cit., p. 124.

11. MELLIER, Denis, op. cit. p. 81-84.

12. VOLPI, Jorge, op. cit., p. 23-26 et p. 177-181. «Lois de la mécanique narrative » et « Lois de la mécanique criminelle », VOLPI, Jorge, op.cit., p. 23-28 et p. 235-241.

13. VOLPI, Jorge, op. cit., p. 23. «Pendant des années, on nous a fait croire que quand nous lisons un roman ou un récit écrit à la première personne [...] personne ne se chargeait de nous conduire par la main jusqu'aux devinettes de la trame [...] C'est par ce biais que l'on se forge l'illusion qu'un livre est un monde parallèle dans lequel on s'aventure pour son plaisir. Rien de plus faux. En ce qui me concerne, j'ai toujours trouvé minables les auteurs qui cherchent à se cacher derrière ce qu'ils écrivent, comme si rien d'eux-mêmes ne filtrait dans leur discours et le choix de leurs mots, et ce à seule fin de nous endormir avec une dose de prétendue objectivité ", VOLPI, Jorge, op. cit., p. 23.

14. Le titre de la première loi, «Toda narración ha sido escrita por un narrador " - «Toute narration a été écrite par un narrateur »-, institue ce jeu à lui seul, en remplaçant le mot « narrée » par « écrite ». VOLPI, Jorge, op. cit., p. 23 dans les deux cas.

15. VOLPI, Jorge, op. cit., p. 24. «(...) le guide qui les conduit à travers du récit [...] qui se risque à guider les pas de ses lecteurs », VOLPI, Jorge, op. cit., p. 24.

16. VOLPI, Jorge, op. cit. p. 25. « la vérité est ma vérité, un point c'est tout », VOLPI, Jorge, op. cit., p. 26.

17. Jorge Volpi, op. cit., p. 179. «Un cadavre, une blessure ou l'échec d'autrui sont les mots, les signes par lesquels le criminel exprime sa vision du monde» et "Celui qui est capable d'assassiner, de voler ou de trahir n'aura de cesse de se justifier et de fixer, dans son but, son critère de vérité au regard de ses actes. [...] Tous les criminels sont obsédés par le bilan de leurs actes, plus encore que ceux qui les pourchassent et cherchent à les punir », VOLPI, Jorge, op. cit., p. 238 et p. 237-238.

18. «Lois de la mécanique criminelle » et « Lois de la mécanique narrative », VOLPI, Jorge, op.cit., p. 23-28 et p. 235-241.

19. DUFLO, Colas, op. cit., p. 123-127.

20. L'anagramme formée par «Links » et «Inge " (la maîtresse de Bacon, dont la véritable identité n'est révélée qu'à la fin du roman), dont la combinaison des noms permet de reconstituer presque le nom «Klingsor», renforce les parallélismes que le mythe de Parsifal établit entre Klingsor et Links et Inge et Kundry, un démon féminin. Pour une étude détaillée des jeux de langage dans ce roman, voir CALDERON, Sara, Jorge Volpi ou l'esthétique de l'ambiguité, Paris, L'Harmattan, 2010, p. 36-67.

21. Agatha Christie, Le meurtre de Roger Ackroyd, Paris, Poche, 1992, p. 220-221.

22. En ce sens, Mellier conclut que le policier est une fiction de la détection informée en réalité par l'abduction, c'est-à-dire, par l'invention d'une règle telle que, si elle était vraie, expliquerait le phénomène. Denis Mellier, op. cit., p. 89-95.

\section{AUTHOR}

\section{SARA CALDERÓN}

Maître de Conférences, Université Nice Sophia Antipolis, LIRCES EA 3159 